\title{
La N-myristoyl transférase, carrefour entre virologie et oncologie : une voie d'accès à des anticancéreux et à des antiviraux d'un genre nouveau?
}

La N-myristoyl transférase (NMT) catalyse l'addition cotraductionnelle d'un résidu myristate sur une glycine aminoterminale de protéines virales et de nombreux produits d'oncogènes. Récemment, des enzymes de levures et de mammifères ont été analysées en détail, et leur ADNc a été cloné. La recherche sur les inhibiteurs de la (ou des) NMT est potentiellement intéressante car de telles substances pourraient spécifiquement inhiber la fonction de protéines oncogéniques et l'assemblage de virus pathogènes, tels le VIH, le virus de l'herpès ou de la leucémie $T$ humaine (HTLV).

\section{Jean A. Boutin}

\section{ADRESSE}

Jean A. Boutin : docteur ès sciences, chef de projet de recherches, division des peptides. Institut de recherches Scrvier, 11, rue des Mouli-

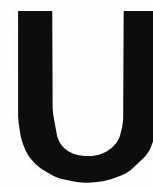

Une étape importante de la maturation des protéines est l'acylation, c'està-dire une modification co- $^{-}$ou post-traductionnelle impliquant un acide gras. Il existe de nombreux types d'acylation: palmitoylation, farnésylation, myristoylation, etc. [1]. La spécificité de ces étapes est souvent très étroite : elles concernent un nombre relativement restreint de protéines présentant des sites d'acylation très précisément définis.

\section{De la myristoylation comme cible thérapeutique}

La myristoylation a été mise en évidence dans les annćes 1980 sur une protéine "endogène ", la protéine kinase dépendante de l'AMP cyclique [2], mais a concerné très rapidement surtout des produits d'oncogènes ou des protéines structurales de virus [3]. Elle est, semble-t-il, un événement plus rare que la palmitoylation mais concerne plus d'espèces différentes de protéines que la farnésylation. D'un point de vue fonctionnel, cette maturation pourrait avoir deux types de rôles (figure 1): permettre une association aspécifique ou spécifique avec une structure membranaire. L'association aspécifique provient du ciblage des protéines myristoylées vers les compartiments les plus hydrophobes de la cellule (membrane plasmique, réticulum endoplasmique), processus classique pour ce qui concerne les acylations 
[1]. L'interaction entre protéines myristoylées et membranes pourrait jouer un rôle dans la fusion des virus avec la membrane plasmique, à l'image des interactions hydrophobes décrites pour la fusion du virus de Sendai avec la membrane des érythrocytes [5]. A. Aderem [6] rappelle, dans une revue récente sur les MARCKS - ces substrats myristoylés parmi les plus importants de la protéine kinase $\mathrm{C}$ - que l'association de ccs protéines avec la membrane plasmique est un processus réversible qui dépend du niveau de phosphorylation de ces protéines: phosphorylées par la protéine kinase $\mathrm{C}$, elles se détachent de la membrane, déphosphorylées au niveau du cytoplasme, elles se réassocient avec la membrane au voisinage de la protéine kinase $\mathrm{C}$ $\left(m / s n^{\circ} 2\right.$, vol. 9, p. 221). Il y a donc, dans certains cas, une réelle réversiblité de l'association protéine myristoylée/membrane. Plus spécifiques seraient les associations des protéines myristoylées avec des structures macromoléculaires telles que le récepteur présomptif de pp60 $0^{s t}$, produit de l'oncogenèse $[7,8]$, ou les protéines $\mathrm{G}^{*}[9,10]$, voire même interférence avec l'association à l'ADN de la protéine FBR (gag-fos)** [11]. Il existe relativement peu de protéines endogènes de la cellule saine qui soient myristoylées (Tableau I). En revanche, deux groupes d'affections sont particulièrement concernées par la myristoylation : celles associées à la surexpression de certains types d'oncogènes, en particulier les tyrosine protéine kinases sans fonction de récepteurs, et celles liées à l'infection d'un organisme sain par des virus, plus particulièrement - mais pas uniquement - les rétrovirus.

En bref, tout cancer surexprimant un produit d'oncogène à fonction tyrosine protéine kinase devrait être sensible à des inhibiteurs de N-myristoyl

\footnotetext{
* Protéines $G()$ : Les protéines $G$ forment une famille de protéines multimériques régulatrices à activité GTPasique. Elles sont impliquées dans la transduction de signaux transmembranaires [60].

** Protéine FBR (gag-fos): FBR est un oncogène du virus d'un ostéosarcome murin (MuSV). Le produit de ce gène est une forme de v-fos qui diffère de Fos aux deux extrémités : à l'extrémité $N$-terminale, 24 acides aminés de fos sont remplacés par les 310 acides aminés de la protéine gag virale, alors que la partie C-terminale contient deux modifications plus complexes [61, 62].
}

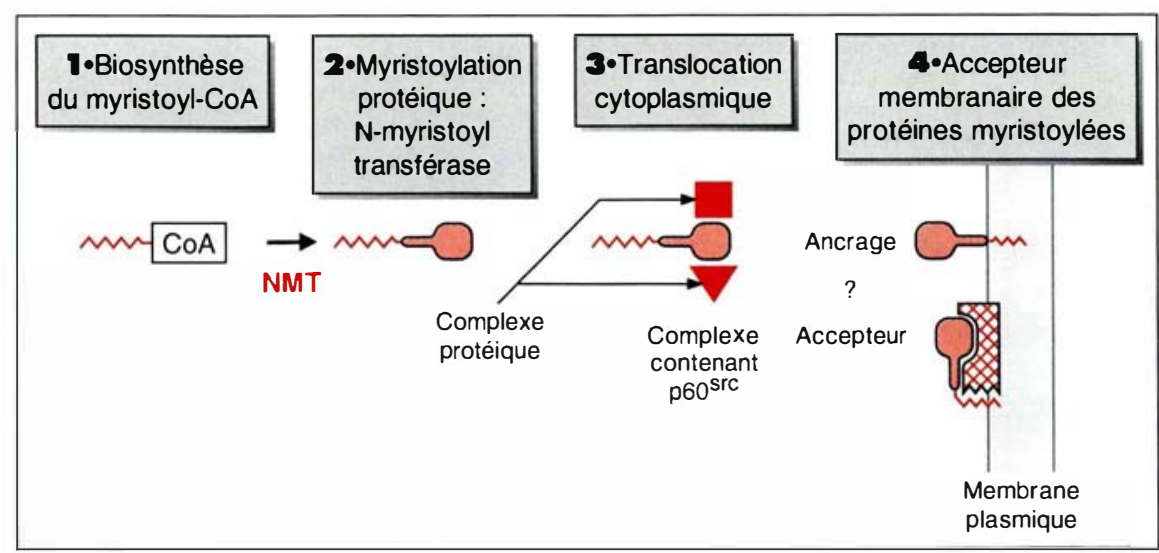

Figure 1. Cibles spécifiques pour l'inhibition de la myristoylation. (1) biosynthèse du myristoyl-CoA, (2) N-myristoyl transférase, (3) translocation cytoplamique, (4) accepteur membranaire des protéines myristoylées. NMT: N-myristoyl transférase. (Adaptée de Felsted et al, [4].)

transférase. En effet, toutes les tyrosine protéine kinases oncogéniques (non liées à des récepteurs, par exemple la famille src, et donc à l'exclusion de récepteurs tyrosine kinases tels que Ros, Neu, Trk, Kit, Sea, Ret ou Met) ([12] pour revue complète) décrits à ce jour dans la littérature sont myristoylées (Tableau II, p. 688). Le rôle prépondérant de la myristoylation dans la capacité transformante de ces tyrosine protéine kinases a été démontré : sans myristoylation, ces produits d'oncogènes sont incapables de transformer une cellule saine. Par exemple, Kamps $e t$ al. [13] montrent que l'absence de myristoylation de la tyrosine protéine kinase $\mathrm{p} 60^{\mathrm{src}}$, n'entraîne en rien une diminution de sa capacité catalytique, mais empêche son association avec un site (probablement spécifique) de la membrane plasmique [7]. Cette tyrosine protéine kinase est alors incapable de phosphoryler ses substrats, eux-mêmes associés à la membrane plasmique puisque, sans myristoylation, pp60 src est très largement localisée au niveau du cytoplasme.

En virologie, les données sont similaires : sans myristoylation, certaines protéines structurales codées par le matériel génétique viral ne peuvent pas s'associer de façon adéquate, rendant les particules virales, ainsi malformées, incapables d'infecter de nouvelles cellules saines. Par exemple, dans le virus de la poliomyélite, l'absence de la glycine N-terminale de la protéine structurale VP4 (mutée en alanine) empêche la formation de particules virales infectieuses [14]. De même, le précurseur $\left(\operatorname{Pr} 55^{a a g}\right)$ des protéines structurales principales du virus d'immunodéficience humain (VIH-1) doit être myristoylé pour permettre une maturation normale de VIH : si la glycine N-terminale de Pr55 $5^{a g}$ est mutée en alanine, l'association stable avec la membrane plasmique de la cellule hôte et l'assemblage des particules de VIH à ce niveau sont impossibles [15]. Ce qui est valable pour les virus de la poliomyélite et du SIDA l'est également pour de nombreux autres types de virus, parmi lesquels: grippe, influenza, HTLV-1, mais aussi virus à $\mathrm{ADN}$, tels que celui de l'hépatite, les herpès et les cytomégalovirus, pour ne citer que les principaux (voir Tableau III, p. 689 pour une liste indicative ainsi que [16] pour des précisions concernant les protéines virales myristoylées).

Sur la base de nombreux travaux sur l'acylation du produit de certains oncogènes et de protéines structurales de nombreux types de virus, il apparaît donc que cibler la myristoylation en tentant d'interférer avec son déroulement pourrait amener de nouvelles approches thérapeutiques pour certains types de cancer et/ou d'infections virales. Trois solutions sont possibles pour atteindre ce but : inhiber la N-myristoyl transférase ou - sur la base de l'idée originale de Heuckeroth et Gordon - fournir à cette enzyme des cosubstrats alternatifs qui 


\section{RÉFÉRENCES}

1. Han KK, Martinage A. Possible relationship between coding recognition amino acid sequence motif or residuc(s) and posttranslational chemical modification of proteins. Int J Biochem 1992; $24:$ 1349-63.

2. Carr SA, Biemann K, Shoji S, et al. NTetradecanoyl is the $\mathrm{NH}_{2}$-terminal blocking group of the catalytic subunit of cyclic AMP-dependent protein kinase from bovinc cardiac muscle. Proc Natl Acad Sci USA $1982 ; 79$ : 6128-31.

3. Schultz AM, Oroszlan S. Myristylation of gag-onc fusion proteins in mammalian transforming retroviruses. Virology 1984; 133 : 431-7.

4. Felsted RL, Goddard C, Glover CJ. Nmyristoyltransferase as a novel molecular target for the design of chemotherapeutic drugs. In : Glanzer RI, cd. Developments in Cancer Chemotherapy, vol. 2. Boca Raton : CRC Press Inc., 1992: 95-115.

5. Hoekstra D, Klappc K, Hoff $\mathrm{H}$, et al. Mechanism of fusion of Sendai virus : rolc of hydrophobic interactions and mobility constraints of viral membranc proteins. $J$ Biol Chem 1989 ; 264 : 6786-92.

6. Aderem A. The MARCKS brothers : a family of protein kinase $\mathrm{C}$ substrates. Cell 1992 ; 71 : 713-6.

7. Resh MD, I ing $\mathrm{H}$. Identification of a $32 \mathrm{~K}$ plasma membrane protein that binds to the myristoylated amino-terminal scquence of p60v-src. Nature $1990 ; 346$ : 84-7.

8. Goddard C, Arnold ST, Fclsted RL. High affinity binding of an $\mathrm{N}$-terminal myristoylated p60src peptidc. J Biol Chem 1989 ; 264 : 15173-6.

9. I inder ME, Pang IH, Duronio RJ, et al. I.ipid modifications of $\mathrm{G}$ protcin subunits. $J$ Biol Chem 1991; 266 : 4654-9.

10. Vanderncut R, Pantaloni C, Nebout I, et al. Mutagenesis of the amino-terminal glycine to alanine in Gs-alpha subunit alters beta-gamma-dependent propertics and decreases adenylcyclase activation. $J$ Biol Chem 1993 ; 268 : 436-41.

11. Kamata N, Jotte RM, Holt JT. Myristylation alter DNA-binding activity and transactivation of FBR (gag-fos) protcin. Mol Cell Biol 1991; 11 : 765-72.

12. Hunter T. Cooperation between oncogenes. Cell 1991; 64 : 249-270.

13. Kamps MP, Buss JE, Sefton BM. Mutation of NH2-terminal glycine of p60src prevents both myristoylation and morphological transformation. Proc Natl Acad Sci USA 1985 ; 82 : 4625-8.

14. Marc D. La myristylation de la protéine de capside VP4 du poliovirus : son rôle dans lc cycle viral. Thèse de doctorat. Paris-VII, 1991.

15. Bryant M, Ratner I. Myristoylationdependent replication and assembly of human immunodeficiency virus 1. Proc Natl Acad Sci USA 1990; 87: 523-7.

16. Harper DR, Gilbert RL. Viral lipoprotcins. Rev Med Virol 1992 ; 2 : 107-115.

17. Resh M. Specific and saturable binding of pp60v-src to plasma membranes: evidence for a myristyl-src receptor. Cell 1989 ;

rendent les protéines myristoylées anormales et inopérantcs, ou, enfin, tenter d'interférer avec les réccpteurs présomptifs des protéines myristoylées $[7,17,18]$. Cependant, ce concept de récepteurs de protéines myristoylées n'est pour l'instant décrit que pour pp60 src [4], et un tcl récepteur n'a pu être mis en évidence pour les protéines MARKCS [19].

\section{Au cour de la maturation, l'enzyme}

Les protéines myristoylées par la $\mathrm{N}$ myristoyl transférase (NMT, E.C. 2.3.1.97) répondent au même critère exclusif : elles possèdent toutes une glycine en position $\mathrm{N}$-terminale [20] sur laquelle le myristate est attaché par une liaison amide. Cette maturation ne doit pas être confondue avec une autre maturation par le myristate, récemment décrite pour le tumor necrosis factor, qui voit son précurseur myristoylé sur une lysine [21]. La myristoylation conccrne donc un acide gras saturé en C14, l'acide myristique, qui existe de façon endogène sous forme de myristoyl-cocnzyme A. C'est cettc cspèce biochimique qui sert de substrat naturel exclusif à la NMT. L'acide myristique est souvent qualifié de rarc in vivo, représentant 1 à $2 \%$ des acides gras cellulaires totaux [22]. La première purification à homogénéité de la NMT a été rapportée en 1987, à partir d'un matéricl biologique abondant, mais dont le choix - la lcvure (Saccharomyces cerevisiae) [23] - est relativement étonnant dans un contexte de cancérologic et de virologie. En effet, la levure ne pcut servir de modèle aux affections citées précédemment qu'à travers la cible moléculairc elle-même (la NMT). Encore faut-il clairement démontrer que cette cible est identique à cellc viséc dans les cellules de mammifères - au niveau de la protéine elle-même, mais aussi au niveau de sa répartition subcellulaire, puis de sa régulation, de sa spécificité vis-àvis de ses substrats protéiques ainsi que de sa spécificité vis-à-vis de son co-substrat - un point capital dans le cas des co-substrats alternatifs (voir plus loin). La facilité avec laquelle la génétique de $S$. cerevisiae peut être étudiée et modifiée montre néanmoins l'intérêt d'avoir choisi cette source biologique. Une étude tout à fait récente du groupe de J. I. Gordon a permis de montrer l'influence que pouvait avoir la modification du gène NMT1 sur la spécificité de l'enzyme. En effet, en altérant la leucine 99 en proline, ces auteurs montrent que parmi les 14 protéines myristoylées de la levure, quelques-unes seulement voient leur niveau de myristoylation diminué [24]. Leur explication est que l'affinité de la protéine pour ses substrats ne serait modifiée que pour certains, en particulier la protéine Gpap1, une protéine $G$ impliquée dans le cycle normal de croissance de la levure [24, 25]. En l'absence de myristoylation de Gpap1, le cycle de la levure est stoppé en phase G1 [25], une observation conforme à l'effet létal de la mutagenèse par insertion du gène NMT1 chez $S$. cerevisiae [26]. L'enzyme issuc de levure possède un mécanisme de cinétique enzymatique $\mathrm{BiBi}^{*}[27,28]$ et est exclusivement cytosolique [29]. Leurs conclusions quant à la compartimentalisation de la NMT montrent que le produit du gène NMT1 de la levure est exclusivement cytosolique dans $S$. cerevisiae alors qu'environ $20 \%$ de l'activité de myristoylation de cette cellule est associée à la fraction membranaire [29]. Cela est la première différence fondamentalc entre l'enzyme de levure et les enzymes de mammifères pour lesquelles une activité microsomiale dépendante des lipides peut être mesurée [30]. En effet, jusqu'à récemment, pratiquement rien n'était connu de NMT issue(s) d'organismes plus complexes, susceptibles de subir une infection virale ou une transformation cancéreuse. Si quelques comparaisons directes ont été entreprises, elles ont été très fragmentaires [31]. Elles ne permettent pas de juger globalement de la transposabilité des propriétés de la NMT de levure aux NMT de cellules de mammifères. Les travaux entrepris par d'autres groupes que celui de J. I. Gordon, ces dernières années,

\footnotetext{
* Une cinétique enzymatique BiBi : Ce type de cinétique enzymatique ordonnée séquentielle $B i$ Bi indique que la NMT fixe le myristoyl-CoA avant le peptide substrat, puis que le Coenzyme $A$ est relargue

avant le peptide myristoylé [27].
} 
ont cn commun qu'ils concernent tous des tissus ou des cellules dc mammifères, csscntiellcment le cerveau (bœuf, rat). Ces travaux commencent à compléter les résultats obtcnus sur la levure, en mettant notamment cn évidence l'existence d'isoformes cytosoliques de la NMT [32, 33]. En utilisant des ccllules cancércuscs de souris (L1210, bicn connucs des cancérologues, car véritables cobayes de la découvertc des cytotoxiques à visée anticancércusc), nous avons confirmé deux points fondamentaux : il y a deux sites d'activité NMT dans cctte cellule, comme d'ailleurs dans le cervcau [34] et dans le foic de rat [35], l'un clairement cytosolique, l'autre microsomial ; il semble y avoir des différences fondamentales dans la spécificité de ces dcux isocnzymes [30].
La purification de l'activité cytosolique conduit à l'obtention de plusieurs espèces enzymatiques dont l'une au moins, purifiće à homogénéité, possède un poids molćculaire plus élevé que la NMT de lcvure (respectivement $67,5 \mathrm{kDa}$ et $52 \mathrm{kDa}$ ) [36]. Sur la spécificité, on retiendra deux faits qui semblent particulièrement étonnants : (1) GNAAAARR, le peptide dérivé de la protéine kinasc $\mathrm{A}$, est le

Tableau I

\section{PROTÉINES MYRISTOYLÉES ENDOGĖNES DE LA CELLULE SAINE}

Protéine kinase AMPc-dépendante

Lipase (précurseur)

NADH cytochrome b5 réductase Calcineurine B

Vinculine

Annexine spécifique de l'intestin (ISA)

Protéine 4.2 de l'érythrocyte

MARCKS(2)

MARCKS-like protein

Créatine kinase flagellaire

Nitric oxide synthase (endothéliale)

Recovérine

Visinine

Neurocalcine

\section{1-kDa calcium-binding protein}

Protéines G

$$
\begin{aligned}
& \text { Gi1 } \alpha \\
& \text { Gi2 } \alpha \\
& \text { Gi3 } \alpha \\
& \text { G0 } \alpha \\
& \text { Gx } \alpha \\
& \text { Gt1 } \alpha \\
& \text { Gt2 } \alpha \\
& \text { G olf } \\
& \text { Gs } \alpha
\end{aligned}
$$

Proto-oncogènes

$$
\begin{aligned}
& c-r e l \\
& c-a b l \text { (type IV) } \\
& c-s r c \\
& c-l c k
\end{aligned}
$$

Carr et al., Proc Natl Acad Sci USA 1982; 79 : 7493-7.

GNHLOISV Komaromy et Sholtz, Proc NatI Acad Sci USA 1987 ; 84: 1526-30.

GAOLSTLG Ozols et al., J Biol Chem 1982; 259 : 13349-54.

GNEASYPL $\quad$ Aitken et al., FEBS Lett $1982 ; 150$ : 314-8.

? ?(1)

GNRHAKAS

GQALGIKS

GAQFSKTA

GSOSSKAP

GCAASSQO

GNLKSVGO

GNSKSGAL

GNSRSSAL

GKONSKLR

Kellie et Wigglesworth, FEBS Lett $1987 ; 213: 428-32$

Wice et Gordon, Cell Biol $1992 ; 116$ : 405-22.

Risinger et al., J Biol Chem $1992 ; 267$ : 5680-5.

Aderem et al., Nature 1988 ; 332: 362-4.

Umekage et Kato, FEBS Lett $1991 ; 286$ : 147-151.

Quest et al., 1992. J Biol Chem 1992; 267 : 15080-5.

Sessa et al., J Biol Chem 1992; 267 : 15274-7.

Dizhoor et al., Science 1991; 251 : 915-8.

Yamagata et al., Neuron 1990; 4 : 469-76.

Okazaki et al., Biochem Biophys Res Comm 1992; 185: 147-53.

GKONSKLA Kuno et al., Biochem Biophys Res Comm 1992 ; 184 : 1219-25.

Mumby et al., (3) Proc Natl Acad Sci USA 1990; 87 : 728-32.

GCTLSAED

GCTVSAED

Jones et Reed, J Biol Chem 1987; 262 : 14241-9.

Itoh et al., Proc Natl Acad Sci USA 1986; 83 : 3776-80.

GCTLSAED

Itoh et al., J Biol Chem 1988; 263 : 6656-64.

van Meurs et al., Proc Natl Acad Sci USA 1987 ; 84 : 3107-11.

GCROSSEE Matsukoa et al., Proc Natl Acad Sci USA 1988; 85 : 5384-8.

Tanabe et al., Nature $1985 ; 315: 242-5$.

Lochrie et al., Science 1985; 228 : 96-9.

Jones et Reed, Science 1989 ; 244 : 790-5.

Itoh et al., Proc Natl Acad Sci USA 1986; 83 : 3776-80.

Brownell et al., Mol Cell Biol $1985 ; 5$ : 2826-31.

Ben-Neriah et al., Cell 1986; 44 : 577-86.

Buss et Sefton, J Virol 1985; $53: 7-12$

Semba et al., Science $1985 ; 227: 1038-40$.

(1) Grâce à des méthodes immunologiques, il est démontré que la vinculine est myristoylée, mais, à notre connaissance, il n'y a pas d'autres informations disponibles sur sa séquence.

(2) Myristoylated alanine-rich kinase C substrate, I'un des substrats principaux de la protéine kinase C (voir revue (6]).

(3) Dans cet article, Mumby et al. démontrent qu'un certain nombre de protéines G incorporent du myristate let certains analogues artificiels) sur leur glycine terminale.

Note: Les protéines indiquées sur cette liste sont celles pour lesquelles il a été démontré qu'elles subissaient cette myristoylation, soit par des méthodes analytiques, soit par des méthodes biochimiques. II subsiste une certaine quantité de protéines pour lesquelles la connaissance de la séquence d'acides aminés laisse prévoir une possible myristoylation. 


\section{RÉFÉRENCES}

18. Goddard C, Arnold ST, Felsted RL. High affinity binding of an N-terminal myristoylated p60src peptide. $J$ Biol Chem 1989 ; 264 : 15173-6.

19. George DJ, Blackshear PJ. Membrane association of the myristoylated alanine-rich C kinase substrate (MARCKS) protein appears to involve myristate-dependent binding in the absence of a myristoyl protein receptor. J Biol Chem 1992 ; 267 : 24879-85. 20. Towler DA, Adams SP, Eubanks SR, et al. Myristoyl CoA: protein Nmyristoyltransferase activities from rat liver and yeast possess overlapping yet distinct peptide substrate specificities. J Biol Chem 1988 ; 263 : 1784-90.

21. Stevenson FT, Bursten SL, Locksley RM, et al. Myristyl acylation of the tumor necrosis factor alpha precursor on specific lysine residues. J Exp Med 1992 ; 176 : 1053-62.

22. Orme TW, McIntyre J, Lynden F, et al. Fatty-acid elongation in a mutant of $S$. cerevisiae deficient in fatty-acid synthetase. Eur $J$ Biochem 1972 ; 24 : 407-15.

23. Towler DA, Eubanks SR, Towery DS, et al. Amino-terminal processing of proteins by N-myristoylation. J Biol Chem 1987 ; 262: $1030-8$.

24. Johnson DR, Duronio RJ, Langner $\mathrm{CA}$, et al. Genetic and biochemical studies of a mutant Saccharomyces cerevisiae myristoylCoA-protein $\mathrm{N}$-myristoyltransferase, NMT72p leu $99-\rightarrow$ pro, that produces temperature-sensitive myristic acid auxotrophy. I Biol Chem 1993; 268 : 483-94.

25. Stone DE, Cole GM, de Barros Lopes $\mathrm{M}$, et al. N-myristoylation is required for function of the pheromone-responsive $G$ alpha protein of yeast : conditional activation of the pheromone response by a temperature-sensitive $\mathrm{N}$-myristoyl transferase. Gene Dev 1991 ; 5: 1969-81.

26. Duronio RJ, Towler DA, Heuckeroth $\mathrm{RO}$, et al. Disruption of the yeast $\mathrm{N}$ myristoyl transferase gene causes recessive lethality. Science 1989; 243 : 796-800.

27. Rudnick DA, McWherter CA, Rocque WJ, et al. Kinetic and structural evidence for a sequential ordered $\mathrm{Bi} \mathrm{Bi}$ mechanism of catalysis by Saccharomyces cerevisiae myristoyl-CoA: protein $\mathrm{N}$-myristoyltransferase. J Biol Chem 1991; 266 : 9732-9. 28. Gordon JI, Duronio RJ, Rudnick DA, et al. Protein N-myristoylation. I Biol Chem 1991; 266 : 8647-50.

29. Knoll LJ, Levy MA, Stahl PD, et al. Analysis of the compartimentalization of myristoyl-CoA : protein $\mathrm{N}$-myristoyltransferase in Saccharomyces cerevisiae. J Biol Chem 1992; 267 : 5366-73.

30. Boutin JA, Clarenc JP, Ferry G, et al. $\mathrm{N}$-myristoyl-transferase activity in cancer cells. Solubilization, specificity and enzymatic inhibition of a N-myristoyl transferase from L1210 microsomes. Eur $J$ Biochem $1991 ; 201: 257-63$.

31. Towler DA, Gordon JI, Adams SP, et al. The biology and enzymology of eukaryotic protein acylation. Annu Rev Biochem $1988 ; 57: 69-99$

32. Glover CJ, Goddard C, Felsted RL. Nmyristoylation of $\mathrm{p} 60$. Identification of a myristoylCoA : glycyl peptide N-myristoyltransferase in rat tissue. Biochem J 1988 ; $250: 485-91$.

\begin{tabular}{|c|c|}
\hline \multicolumn{2}{|c|}{$\begin{array}{c}\text { Tableau ॥ } \\
\text { QUELQUES EXEMPLES } \\
\text { DE TYROSINE PROTÉINE KINASES } \\
\text { ET DE PROTÉINES } \\
\text { ONCOGÉNIQUES MYRISTOYLÉES }\end{array}$} \\
\hline $\begin{array}{l}\text { Tyk-2 } \\
\text { Stk } \\
\text { Lck } \\
\text { Fyn } \\
\text { Hck } \\
\text { Yes } \\
\text { Lyn } \\
\text { Fgr } \\
\text { Ltk } \\
\text { APK1 } \\
\text { Int-1 } \\
\text { Int-2 }\end{array}$ & 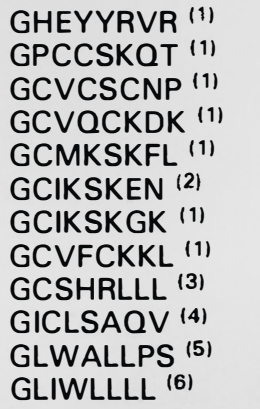 \\
\hline
\end{tabular}

(1) Myristoylée in vitro par la NMT cytosolique de mammifère [36].

(2) Non myristoylée in vitro par la NMT cytosolique de L1210 [36].

(3) Snijders et al., Oncogene 1993; $8: 27-35$

(4) Hirayama et Oko, Plant Mol Biol, $1992 ; 20: 653-62$.

(5) Fung et al., Mol Cell Biol, 1985; 5 : 3337-44.

(6) Moore et al., EMBO J, $1986 ; 5: 919-24$.

Note : Rappelons, d'après Schultz et Oroszlan [3], que de nombreux oncogènes d'origine virale sont exprimés dans les cellules infectées sous forme de protéines de fusion gag-onc, dans lesquelles la protéine de structure gag est myristoylable.

meilleur substrat de l'enzyme de levure, parmi la centaine que le groupe de J. I. Gordon a testés [31]. A contrario, pour les activités cytosolique et microsomiale de mammifères, de nombreux peptides sont meilleurs substrats que GNAAAARR $[30,36]$; (2), par ailleurs, GNAAAARR n'est myristoylé que par la fraction microsomiale de cellules de mammiferes. Aucun des cytosols que nous avons testés (entre autres provenant des lignées cellulaires HL60, L1210, BRL, CEM-CCRF, PSJ, etc.) n'est capable de myristoyler ce peptide [30, 36]. Cette nouvelle différence entre les activités microsomiale et cytosolique ainsi qu'entre les activités cytosoliques de mammifères et de levure souligne encore la nature probablement différente des NMT isolées à partir de ces deux types d'organismes.

Duronio et al, [26] ont cloné l'enzyme de levure, puis l'ont exprimée dans plusieurs systèmes, y compris $E$. coli, elle-même dépourvue de NMT [28]. Le plus surprenant reste pour l'observateur que, malgré ces importants travaux abondamment publiés et repris, ces auteurs n'ont apparem- ment pas réussi à cloner le gène de la NMT dans un organisme plus complexe que $S$. cerevisiae ou $C$. albicans [37]. En effet, bien que, dès 1989, ils aient eu en leur possession le gène complet de la levure, il faudra attendre 1992, et une technique très sophistiquée de rescuing utilisant la complémentarité entre les activités NMT de HepG2 (un hépatocarcinome humain) et une levure artificiellement dépourvue de NMT, pour voir publier des informations sur le gène humain correspondant [38]. L'homologie est de $44 \%$ entre le gène humain et celui de la levure, et, surtout, le gène est unique dans le génome humain [38]. Les NMT cytosoliques et microsomiale(s) chez les mammifères seraient donc issues d'un même et unique gène [38]. Ce résultat nous semble quelque peu en contradiction avec nos propres études [30], où nous avons montré que la NMT microsomiale n'est pas simplement associée à la membrane microsomiale (protéine membranaire extrinsèque ou périphérique [39]) mais en fait partie intégrante (protéine membranaire intrinsèque, intégrale ou encore cryptique [39]), entre 
autres parce qu'elle est activable et solubilisable par les détergents [30]. Il serait unique, à notre connaissance, qu'une protéine issue d'un même gène pût être à la fois cytosolique et intégrée dans la membrane microsomiale. On ne confondra pas, dans cette affirmation, des protéines qui, comme la protéine kinase A, la NADH cytochrome b5 réductase ou p60 c-src peuvent être réparties dans le cytosol et associées à un compartiment membranaire alors qu'issues, évidemment, de gènes communs.

Comme nous l'avons souligné plus haut, il faut savoir laquelle ou lesquelles de ces NMT jouent un rôle dans l'acylation co-traductionnelle de protéines virales (la traduction est cytosolique pour le virus de l'her- pès [40] et le coronavirus [41] mais probablement microsomial pour VIH et pour les produits d'oncogènes), de façon à en connaître précisément les spécificités, leur sensiblilité aux inhibiteurs... Grâce à des systèmes de coexpression dans $E$. coli dépourvus de NMT, il est maintenant possible d'exprimer la NMT et l'un ou plusieurs de ses substrats $[24,42]$, ce qui

Tableau III

\section{VIRUS PRÉSENTANT UNE OU PLUSIEURS PROTÉINES MYRISTOYLÉES}

\begin{tabular}{|c|c|c|}
\hline \multicolumn{3}{|c|}{ Myristoylation essentielle au pouvoir infectant } \\
\hline $\begin{array}{l}\text { Polyoma virus } \\
\text { Simian virus } 40 \\
\text { Duck hepatitis virus } \\
\text { Poliomyelitis virus } \\
\text { Rhinovirus } \\
\text { Reovirus } \\
\text { Varicella-zoster virus } \\
\text { Human immunodeficiency virus (gag) } \\
\text { Human immunodeficiency virus (nef) } \\
\text { Human T Lymphocyte virus } 1 \\
\text { Moloney murine leukemia virus } \\
\text { Mason-Pfizer monkey virus } \\
\text { Spleen necrosis virus }\end{array}$ & $\begin{array}{l}\text { Papovaviridae } \\
\text { Papovaviridae } \\
\text { Picornaviridae } \\
\text { Picornaviridae } \\
\text { Picornaviridae } \\
\text { Reoviridae } \\
\text { Herpesviridae } \\
\text { Retroviridae } \\
\\
\text { Retroviridae } \\
\text { Retroviridae } \\
\text { Retroviridae } \\
\text { Retroviridae? }\end{array}$ & $\begin{array}{l}\text { Krauzewicz et al., J Virol } 1990 ; 64: 4414-20 . \\
\text { Streuli et Griffin, Nature } 1987 ; 326: 619-22 . \\
\text { Macrae et al., Virology } 1991 ; 181: 359-63 . \\
\text { Marc et al., EMBO J } 1989 ; 8: 2661-8 . \\
\text { Chow et al., Nature } 1987 ; 327: 482-6 . \\
\text { Nibert et al., J Virol } 1991 ; 65: 1960-7 . \\
\text { Namazue et al., Intervirol } 1989 ; 30: 268-77 . \\
\text { Bryant et Ratner, Proc Natl Acad Sci USA } 1990 \\
87: 523-7 . \\
\text { Yu et Felsted, Virology } 1992 ; 187: 46-55 . \\
\text { Shoji et al., J Biochem } 1988 ; 103: 747-9 . \\
\text { Schultz et Rein, J Virol } 1989 ; 63: 2370-3 . \\
\text { Rhee et Hunter, J Virol } 1990 ; 64: 4383-9 . \\
\text { Weaver et Panganiban, J Virol } 1990 ; 64 \\
\text { 3995-4001. }\end{array}$ \\
\hline
\end{tabular}

Rous sarcoma virus

Retroviridae

Erdie et Wills, J Virol $1990 ; 64$ : 5204-8.

\section{Myristoylation existante, rôle indéterminé*}

Budgerigar fledging disease virus Hepatitis $B$ virus

Foot-and-mouth disease virus Influenza virus

Vaccinia virus

Rotavirus

Herpes simplex virus

Sendai virus

Bovine leukemia virus

Feline sarcoma virus

Baboon endogenous virus

Mouse mammary tumor virus

Human T lymphocyte virus 2

Murine leukemia virus

Simian immunodeficiency virus

Papovaviridae
Hepadnaviridae
Picornaviridae
Orthomyxoviridae
Poxviridae
Reoviridae
Herpesviridae
Retroviridae
Retroviridae
Retroviridae
Retroviridae
Retroviridae
Retroviridae
Retroviridae
Retroviridae

Schmidt et al., J Virol $1989 ; 63$ : 429-31.

Persing et al., J Virol 1987; 61 : 1672-7.

Belsham et al., J Gen Virol 1991 ; 72 : 747-51.

Hiebert et al., Virology $1986 ; 155$ : 747-51.

Franke et al., J Virol 1989 ; 63 : 4285-91.

Clark et Desselberger, J Gen Virol 1988; 69 : 2681-6.

Maclean et al., J Gen Virol 1989 ; 70 : 3147-57.

Tsai et al., Virology 1985 ; 140 : 289-312.

Rice et al., Virology $1985 ; 142$ : 357-77.

Hampe et al., Cell $1982 ; 30: 775-85$.

Tamura, J Virol $1983 ; 47$ : 137-45.

Schultz et Oroszlan, J Virol 1983 ; 46 : 355-61.

Ootsyama et al., Jpn J Cnacer Res 1985; 76 :

1132-5.

Oroszlan et al., Proc Natl Acad Sci USA 1978 ;

$75: 1404-8$

Henderson et al., J Virol 1988 ; 62 : 2587-95.

* Si le rôle de la myristoylation des protéines Gag des rétrovirus inscrits dans cette partie du tableau n'a pas été étudié spécifiquement, la plupart des auteurs s'autorisent à dire que, par analogie avec ce qui est connu sur d'autres rétrovirus, la myristoylation de Gag est essentielle au pouvoir infectant des rétrovirus. 


\section{RÉFÉRENCES}

33. King MJ, Sharma RK. Demonstration of multiple forms of bovine brain myristoyl CoA : protein $\mathrm{N}$-myristoyl transf crase. Mol Cell Biochem $1992 ; 113$ : 77-81.

34. McIlhincy RAJ, McGlone K. Characterization of a myristoyl CoA : glycylpcptide $\mathrm{N}$-myristoyl transferase activity in rat brain : subcellular and regional distribution. J Neurochem $1990 ; 54: 110-7$.

35. King MJ, Pugazhenthi S, Khandelwal $\mathrm{RL}$, et al. Elcvated N-myristoyl transferasc activity is reversed by sodium orthovanadate in streptozotocin-induced diabetic rat. Biochim Biophys Acta 1993; 1165 : 259-62.

36. Boutin JA, Ferry G, Ernould AP, et al. $\mathrm{N}$-myristoyl-transferase activity in cancer cells. Purification and characterization of a cytosolic isoform from the murine lcukemia cell line L1210. Eur J Biochem 1993 (sous presse).

37. Wiegand RC, Carr C, Minncrly JC, et al. The Candida albicans myristoyl-CoA : protein N-myristoyltransf crasc. J Biol Chem 1992 ; 267 : 8591-8

38. Duronio RJ, Reed SI, Gordon JI. Mutations of human myristoyl-CoA : protein N-myristoyltransf crase causc tempcrature-sensitive myristic acid auxotrophy in Saccharomyces cerevisiae. Proc Natl Acad Sci USA 1992 ; 89 : 4129-33.

39. Tanford C, Reynolds JA. Characterization of membrane proteins in detergent solutions. Biochim Biophys Acta 1976; 457 : 133-70.

40. Hull R, Brown F, Payne C cds. Viro$\log y$, I.ondon and Basingstroke, Macmillan Ref erence Books, 1989.

41. Lai MMC. Coronavirus : organization, replication and cxpression of genome. Annu Rev Microbiol 1990; 44 : 303-33.

42. Duronio RJ, Jackson-Machelski E, Heuckcroth RO, et al. Protein N-myristoylation in Escherichia coli : reconstitution of a cukaryotic protein modification in bacteria. Proc Natl Acad Sci USA 1990; 87 : 1506-10

43. Burnettc B, Kahn R, Glover CJ, et al. Bacterial expression, purification and in vitro N-myristoylation of HIV-1 p17gag. Prot Express Purif $1992 ; 3$ : 395-402.

44. Hcuckeroth RO, Jackson-Machelski E, Adams SP et al. Novel fatty acyl substrates for myristoyl-CoA : protcin N-myristoyl transferase. J Lipid Res 1990; 31 : 1121-9. 45. Kishore NS, Lu T, Knoll LT, et al. The substrate spccificity of Saccharomyces cerevisiae myristoyl-CoA: protcin Nmyristoyltransferase. J Biol Chem 1991 ; 266 : 8835-55

46. Devadas B, Lu T, Katoh A, et al. Substrate specificity of Saccharomyces cerevisiae myristoyl-CoA : protcin N-myristoyltransferasc. J Biol Chem 1992 ; 267 : 7224-39. 47. Rudnick DA, Lu T, Jackson-Malchclski $\mathrm{E}$, et al. Analogs of palmitoyl-CoA that arc substrates for myristoyl-CoA : protein $\mathrm{N}$ myristoyltransferase. Proc Natl Acad Sci USA 1992 ; 89 : 10507-11.

48. Johnson DR, Cox AD, Solski PA, et al. Functional analysis of protcin N-myristoylation: metabolic labclling studics using three oxygen-substitutes analogs of myristic acid and cultured mammalian provide evidence for protein-sequence-spccific incorporation and analog-specific redistribution. Proc Natl Acad Sci USA 1990 ; 87 : 8511-5. permet d'étudier la spécificité de cette NMT. Dès que des NMT d'autres origines auront étć clonées, il sera possible de comparer lcur spécificité dans ces systèmes. Altcrnativement, une protéine substrat rccombinante pourra ctre produite sous forme non acylée dans un système d'cxpression dépourvu de NMT (E. coli, par excmplc). Dès lors, ccttc protéine, après purification, pourra ĉtrc incubéc avcc les compartiments subccllulaircs microsomiaux ou cytosoliques [43], pcrmettant ainsi de mieux comprendrc les rôles respcctifs des NMT associées à ces deux compartiments.

\section{La notion de substrats alternatifs de la NMT}

Il est classique, en pharmacologie moléculaire, d'étudier une cnzyme cible afin d'en découvrir des inhibiteurs que l'on espère être ensuite capables de reconnaître cettc cible dans un milieu biologique plus complexe tel que la ccllule ou, a fortiori, l'organisme. Heuckeroth et al. posent unc nouvclle question fort originalc cst-il possible de fournir à la NMT (puis, plus tard, à la cellule ou à l'organismc) un composć susccptible d'être pris pour du myristatc, mais sans en avoir toutes lcs caractéristiques biophysiques. Les caractéristiques conférées à la protéine par le myristatc permettent à cettc protéinc acylée dc s'associer à un récepteur [7], à un autre complexc protéiquc [9], à unc séquence particulière de l'ADN [11] ou simplement à un autre site hydrophobe (membrane plasmique, par cxemple). En utilisant un pseudo-myristate, on pcut altérer lcs constantes d'association des complexes ci-dcssus. Pour cc faire, trois éléments sont néccssaires: (1) lc substrat altcrnatif doit ĉtre suffisamment voisin du myristate pour être reconnu par l'acyl transférasc qui va lc transformer en dérivé du coenzyme A et donner le substrat de la NMT sous forme de "substrat-alternatifCoA "; (2) cc substrat alternatif doit être reconnu par la NMT ; et enfin (3), les caractéristiques de la protéine ainsi acyléc doivent être suffisamment altérées pour que les associations mentionnćes plus haut ne puissent pas se fairc. On obtient dès lors un moyen d'interagir avec la myristoy- lation sans l'inhiber. Très tôt dans leurs études sur la NMT de levure, Heuckcroth et al. sc sont intércssés aux spécificités (substrats et cosubstrats) de cettc cnzymc. En particulicr, ils ont montré quc, bicn que la NMT de levure ne reconnaisse pratiquement que lc myristate comme co-substrat naturel, son substrat étant exclusivement la glycine $\mathrm{N}$-terminale d'une protéinc, ellc est capable de reconnaîtrc des co-substrats artificicls dérivés du myristate, en particulier des composés linéaires dans lesquels un méthylène est remplacé par un hétéro-atomc $(\mathrm{O}, \mathrm{S})$ [44-46]. Ils ont même synthétisé des composés finalement plus proches d'une structure de palmitate que dc celle du myristate [47]. Plus d'une containe de structures de ce type ont étć synthétisécs puis tcstées sur divcrs types de modèles. Quclques-uns de ces analogues montrent une activité très intércssante cn pharmacologie antiviralc. En cffct, les protéincs cndogènes acylćes par ces composés artificiels, qui sont plus polaires que le myristate, ne sont pas distribućcs normalement dans la cellule [48] et voient souvent leur fonction altéréc, non pas à travers une inhibition dc lcur capacité catalytique - quand elle cst connuc - mais à causc d'une mauvaise distribution subcellulaire. J. I. Gordon et al. ont multiplić les expéricnces démontrant que ces substrats alternatifs remplacent bien le myristate endogène dans ses fonctions cellulaires d'acylation. En particulier, nous citerons les trois cxcmples suivants : l'intégration du 10-propoxydécanoyl-CoA ne modific pas la distribution subcellulairc de la plupart des protéines myristoylées dans BC3H1 (unc lignéc cellulaire murine) à l'exception de celle de pp60sc et d'une protéine myristoylée de $63 \mathrm{kDa}$ [49] ; l'incubation de ccllules H9 infectées par VIH-1 (souche HXB2gptX) avec de l'acide 12-méthoxydodécanoïque entraîne la diminution de la formation de syncytiums, du niveau de p24 et de l'activité transcriptase inverse, alors que l'acide 5-octyloxypentanoïque inhibc l'asscmblage du virus lcucémique murin de Moloncy (MoMuLV), test sur lequcl l'acidc 12-méthoxydodécanoïque est quant à lui inactif [50] ; l'incorporation de l'acide 12- 
méthoxydodécanoïque dans le précurseur Pr55 gag de VIH-1 inhibe sa maturation protéolytique et la production de virus dans une lignée cellulaire lymphoïde humaine infectée chroniquement [51]. Il est à noter également deux nouvelles approches thérapeutiques ouvcrtes par l'activité de certains de ces composés sur la viabilité des trypanosomes [52] ainsi que de C. albicans [37].

\section{Les inhibiteurs de NMT: antiviraux et anticancéreux}

Il s'agit bien de montrer ici au lccteur l'intérêt que les NMT peuvent présenter en tant que cibles thérapcutiques potentielles. La tendance de ces dernières années, qui est d'essayer de faire profiter le patient des extraordinaircs progrès des connaissances sur la transformation cellulaire et sur la virologie - particulièrement la rétrovirologie -, est en passe d'aboutir à la multiplication de programmes de recherche en oncologie moléculaire et en virologic, à la fois publics et privés. Les voies les plus explorées ont été ccs dernières années les tyrosine protéinc kinases avec la découverte encourageante des tyrphostines, ces inhibiteurs "spécifiques " de ces enzymes, et la Sfarnésyl transférase dont des inhibiteurs pourraient être actifs dans les cancers surexprimant l'oncogène ras $[53,54]$. Le cas de la Nmyristoylation semble tout à fait extraordinaire. La NMT joue un rôle central non seulement dans certaines maladies cancéreuses, mais aussi dans certaines affections virales - certainement parmi les principaux fléaux de notre civilisation moderne ! Ses inhibiteurs pourraient nous donner accès à des composés qui, à l'inverse des inhibiteurs de protéases virales ou de transcriptases inverses rétrovirales, ne seraient pas spécifiques d'un type de virus, mais bien de nombreux virus à myristate (Tableau III). Cela ne doit pas faire perdre de vue l'accumulation nécessaire de données pour l'obtention de telles molécules actives. En janvier 1993, il n'existe aucune étude systématiquc publiée sur des inhibiteurs de NMT. Quatrc types d'inhibiteurs ont été décrits : les octapeptides [20], les analogues de myristoyl-CoA $[55,56]$ et les analo$\begin{array}{lllll}m / s & n^{\circ} & 6-7 & \text { vol. } 9 \text {, juin-juillet } 93\end{array}$

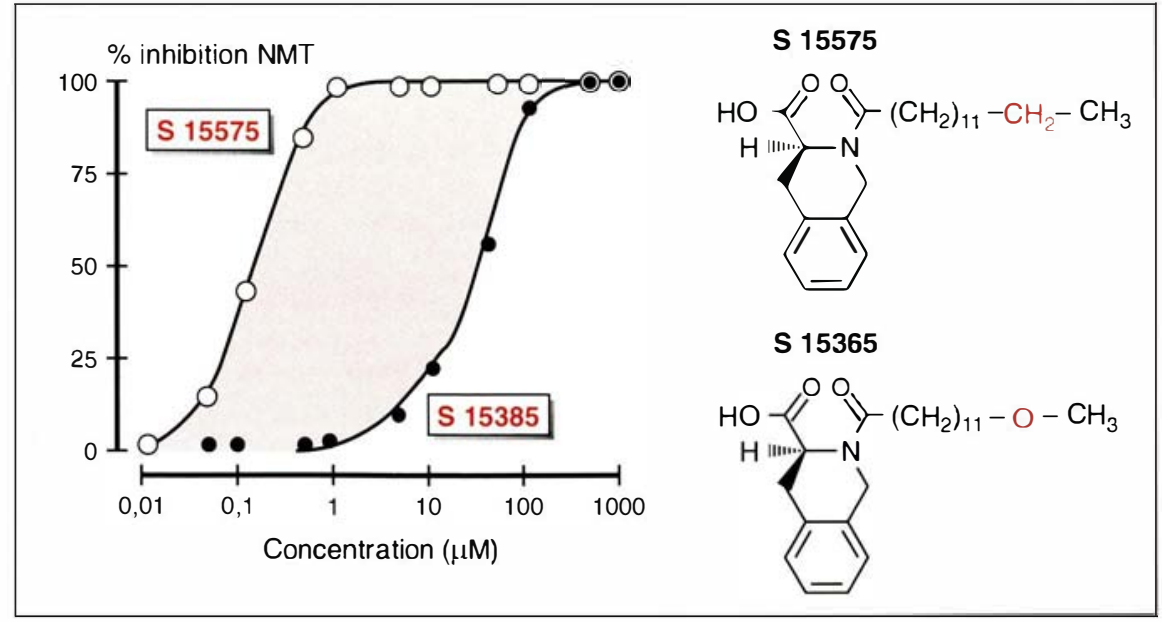

Figure 2. Comparaison de la capacité d'inhibition de la NMT par deux "myraas ". S 15575, le plus puissant des "myraas " que nous ayons synthétisés à ce jour est ici comparé, sur le même test, au $S$ 15365. Les deux composés ne diffèrent que par la présence d'un oxygène en position 13 (S 15365). (Adaptée de [36].)

gues de myristoylglycine [57]. Très brièvement, il semble que ces composés, qui sont d'excellents outils pharmacologiques pour l'étude des NMT, possèdent en tant qu'agents pharmacologiques une au moins des propriétés rédhibitoires suivantes : (1) instabilité plasmatique potentielle des octapeptides du type GLAAAARR ainsi que, peut-être des problèmes de pharmacologie cellulaire (difficulté de la pénétration de ces produits dans la ccllulc cible) tant qu'ils n'auront pas été modifiés en pseudo-peptides [58] ; (2) complexité de synthèse des analogues de coenzyme A - avec peut-être des problèmes de spécificité de la cible puisque la partie coenzyme est susceptible d'être reconnue par d'autres enzymes; (3) hyper-réactivité des dérivés aldéhydes de la myristoylglycine dont la durée de vie plasmatique est toujours très courte. Nous avons synthétisé une gamme de dérivés de myristoylaminoacides, les "myraas" [Boutin et al., en préparation], dont certains sont des inhibiteurs puissants de NMT de mammifères, avec des IC50 allant jusqu'à $10^{-7} \mathrm{M}$ [36]. L'une des constatations les plus troublantes quc nous ayons faites est que la substitution de la partie myristoylée du meilleur inhibiteur, le S 15575 , par un analogue du myristate tel que ceux décrits par le groupe de J. I. Gordon, fait perdre à cette molécule pratiquement toute sa capacité d'inhiber l'activité NMT cytosolique (divisée par 1000, figure 2). Cette observation semble suggérer que ces analogues d'acide myristique ne sont pas reconnus par la ou les NMT cytosolique(s) de cellules L1210, ou du moins qu'ils ne sont pas reconnus avec la même affinité. Les premiers résultats sont toutefois troublants: l'action de nos inhibiteurs est peu importante sur le cycle du virus de la poliomyélite. Ils ne protègent pas les lymphocytes $\mathrm{T}$ en culture de la cytopathie engendrée par le VIH. Et s'ils inhibent fortement l'infection de cellules saines par les virus de l'Herpes simplex 1 et 2, ces données ne semblent pas être retrouvées in vivo. Certains sont actifs sur la phase tardive d'infection dans un test décrit par Savatier et al. [59] utilisant des cellules transcomplémentantes (buffalo rat liver) infectées par un rétrovirus murin recombinant (MoMuLV SVnlsLacZ). Les "myraas" sont des composés lipoylés, et donc à caractère fortement hydrophobe. Leur pharmacologie cellulaire n'a pas été encore suffisamment étudiée.

\section{Moins qu'une conclusion, une perspective}

Notre découverte d'inhibiteurs puissants de la NMT cytosolique est un 


\section{RÉFÉRENCES}

49. Heuckeroth RO, Gordon JI. Altercd membrane association of p60v-src and a murine $63-\mathrm{kDa} \mathrm{N}$-myristoyl protein after incorporation of an oxygen-substituted analog of myristic acid. Proc Natl Acad Sci USA 1989 ; 86 : 5262-6.

50. Bryant ML, Heuckeroth RO, Kimata $\mathrm{JT}$, et al. Replication of human immunodeficiency virus 1 and Moloney murine leukemia virus is inhibited by different heteroatom-containing analogs of myristic acid. Proc Natl Acad Sci USA 1989; 86 : 8655-9.

51. Bryant ML, Ratner L, Duronio RJ, et al. Incorporation of 12-methoxydodecanoate into the human immunodeficiency virus 1 gag polyprotein processing and virus production in a chronically infected human lymphoid cell line. Proc Natl Acad Sci USA 1991; 88 : 2055-9.

52. Doering TL, Raper J, Buxbaum LU, et al. An analog of myristic acid with selective toxicity for African trypanosomes. Science 1991 ; 252 : 1851-4.

53. Khosravi-Far R, Cox AD, Kato $\mathrm{K}$ et al. Protein prenylation : key to ras function and cancer intervention? Cell Growth Diff $1992 ; 3: 461-9$.

54. Hara M, Akasaka K, Akinaga S et al. Identification of Ras farnesyltransferase inhibitors by microbial screening. Proc Natl Acad Sci USA 1993 ; 90 : 2281-5

55. Glover CJ, Tellez MR, Guziec FS, et al. Synthesis and characterization of inhibitors of myristoyl-CoA : protcin Nmyristoyltransferase. Biochem Pharmacol 1991 ; 41: 1067-74.

56. Paige LA, Zheng GQ, DeFrecs SA, et al. S-(2-oxopentyadecyl)-CoA, a nonhydrolyzable analoguc of myristoyl-CoA, is a potent inhibitor of myristoyl-CoA : protein $\mathrm{N}$ myristoyltransferase. J Med Chem 1989 ; 32 : 1665-8.

57. Tashiro A, Shoji S, et Kubota Y. Antimyristoylation of the gag proteins in the human immunodeficiency virus-infected cells with $\mathrm{N}$-myristoyl glycinal diethylacetal resulted in inhibition of virus production. Biochem Biophys Res Commun 1989; 165 : 1145-54.

58. Fauchère JL. Elements for the rational design of peptide drugs. Adv Drug Res 1986 ; 15:29-69.

59. Savatier N, Rocancourt D, Bonncrot C, et al. A novel system for screening antiretroviral agents. J Virol Meth $1989 ; 26$ 229-36.

60. Bourne ER, Sanders DA et McCormick F. The GTPase superfamily : a conserved switch for diverse cell functions. Nature $1990 ; 348: 125-32$.

61. Jenuwein $\mathrm{T}$, Müller D, Curran $\mathrm{T}$ et al. Extended life span and tumorigenicity of nonestableshed mouse connective tissue cells transformed by the fos oncogene of FBR MuSV. Cell $1985 ; 41$ : 629-37.

62. Van Van Beveren C, Van Straaten F Curran T et al. FBR murine osteosarcoma virus. II. Nucleotide sequence of the provirus reveals that the genome contains scquences acquired from two cellular genes. Viro- pas vers l'obtention de composés de référence dans la recherche de ces antiviraux et anticancéreux potentiels. Il reste clairement une importante série de problèmes à résoudre, à la fois fondamentaux et pharmacologiques : (1) Quels sont les rôles des NMT microsomiales et cytosoliques? Leurs spécificités sont-elles différentes? Le site de néo-synthèse des protéines virales ou oncogéniques (réticulum endoplasmique ou cytosol) détermine-t-il à lui seul le site de maturation, puisque cette étape est co-traductionnelle? Peuvent-elles reconnaître avec efficacité les analogues d'acide myristique ? (2) Y a-til une régulation de l'activité et/ou de l'expression de ces différentes NMT due à la situation pathologique ; une sorte d'induction ou de régulation produite par les carcinogènes, peutêtre à travers le pool de protéines induites par ces composés ou lors de la dédifférenciation? Cette régulation - ou un mécanisme voisin pourrait-elle aussi êtrc liée à l'expression de certaines des protéines virales? (3) Peut-on raisonnablement penser obtenir des inhibiteurs spécifiques de l'une des NMT compartimentées? Quelles seront les interférences de ces inhibiteurs avec la myristoylation des protéines endogènes - c'est-à-dire celles faisant partie du métabolisme normal de la cellule saine (voir liste non exhaustive, Tableau I) ? Ceux que nous avons découverts pourraient préfigurer des dérivés dont la pénétration cellulaire serait améliorée tout en gardant une toxicité globale qui semble faible chez la souris $(>200 \mathrm{mg} / \mathrm{kg}$, i.p.)

\section{Remerciements}

Je tiens tout particulièrement à remercier Ron Felsted, NCI, Chris Potter, Sheffield University, Sylvie van der Werf, Institut Pasteur, et Jean-François Nicolas, Institut Pasteur, pour nos discussions encourageantes sur l'évolution de ces programmes NMT ; Jean-François Prost et Michel Vincent, IdRS, pour leur intérêt pour ces recherches, et enfin Gilles Ferry, Jean-Pierre Clarenc et Anne-Pascale Ernould pour leur implication dans ces programmes. Merci à Catherine Séchépée pour son aide dans la réalisation des figures et à Bénédicte Antoine pour sa relecture critique du manuscrit.

\section{Summary}

$\mathbf{N}$-myristoyl transferase, at the junction of virology and oncology : a new approach towards antiviral and antioncotic drugs?

N-myristoyl transferase (NMT, E.C. 2.3.1.97) catalyzes a $\mathrm{CO}^{-}$ translational modification present in two groups of proteins, viral and oncogenic. The yeast enzyme has been thoroughly studied: it has been purified, characterized, its gene cloned and expressed in yeast and $E$. coli. Several ways of modulation of its activity have been described. Because this enzyme, as a target, could represent a major breakthrough in antiviral as well as antioncogenic therapy, it is of major importance that the NMT activity be fully studied in mammalian systems. The first experiments, including the purification of a cytosolic isoform, suggest that some huge differences exist between NMTs from various sources, unlike what had been suggested in the literature so far. The present review both deals with some of these differences and comprehensively reviews the proteins that are myristoylated. It also presents the various inhibitors of NMT described so far. New inhibitors of NMT could become new classes of antiviral and/or anticancer compounds.

\section{TIRÉS A PART}

\section{J. A. Boutin.}

